\title{
An Atkinson-type theorem for B-Fredholm operators
}

\author{
by \\ M. Berkani (Oujda) and M. Sarih (Kénitra)
}

\begin{abstract}
Let $X$ be a Banach space and let $T$ be a bounded linear operator acting on $X$. Atkinson's well known theorem says that $T$ is a Fredholm operator if and only if its projection in the algebra $L(X) / F_{0}(X)$ is invertible, where $F_{0}(X)$ is the ideal of finite rank operators in the algebra $L(X)$ of bounded linear operators acting on $X$. In the main result of this paper we establish an Atkinson-type theorem for B-Fredholm operators. More precisely we prove that $T$ is a B-Fredholm operator if and only if its projection in the algebra $L(X) / F_{0}(X)$ is Drazin invertible. We also show that the set of Drazin invertible elements in an algebra $A$ with a unit is a regularity in the sense defined by Kordula and Müller [8].
\end{abstract}

1. Introduction. Let $A$ be an algebra with a unit $e$. An element $x$ of $A$ is called regular if there is an element $b$ of $A$ such that $x b x=x$. In this case $b$ is called a generalized inverse of $x$. Following [10] we say that an element $x$ of $A$ is Drazin invertible of degree $k$ if there is an element $b$ of $A$ such that

$$
x^{k} b x=x^{k}, \quad b x b=b, \quad x b=b x .
$$

Recall that the concept of Drazin invertibility was originally considered by M. P. Drazin in [5] where elements satisfying (1) are called pseudo-invertible elements.

In [10] an element $x$ of $A$ satisfying (1) for $k=1$ is called group invertible. It follows from [11, Theorem 3.3 and Proposition 3.9] that an element of $A$ is group invertible if and only if it has a commuting generalized inverse. In [7] and [11] group invertible elements are called respectively simply polar elements and "generalized invertible" elements. It follows also from [11, Theorem 3.3 and Proposition 3.9] that an element $x$ of $A$ is group invertible if and only if there is a generalized inverse $b$ of $x$ such that $e-x b-b x$ is invertible in the algebra $A$.

In the first part of this paper we show that the set of Drazin invertible elements in the algebra $A$ is a regularity in the sense of Kordula and Müller (Definition 2.2).

2000 Mathematics Subject Classification: 16U99, 47A10, 47A53. 
So if $A$ is a Banach algebra with unit, the spectrum associated to this regularity satisfies the spectral mapping theorem. We also show that if $a$ is a Drazin invertible element in a Banach algebra $A$ and if $b$ is small in norm and is an invertible element of $A$ commuting with $a$, then $a+b$ is invertible. Moreover we show that the product of two commuting Drazin invertible elements is also Drazin invertible.

In the second part of this paper we consider a Banach space $X$ and the Banach algebra $L(X)$ of bounded linear operators acting on $X$. In Theorem 3.4 we show that an operator $T \in L(X)$ is a B-Fredholm operator if and only if its projection in the algebra $L(X) / F_{0}(X)$ is Drazin invertible, where $F_{0}(X)$ is the ideal of finite rank operators in the algebra $L(X)$. As an application we show that the set of B-Fredholm operators is stable under finite rank perturbations, and we prove that the product of two commuting B-Fredholm operators is a B-Fredholm operator. At the end of this paper, we give two open questions.

2. On Drazin invertibility. Let $X$ be a vector space and let $T$ be a linear operator acting on $X$.

Definition 2.1. For $n \in \mathbb{N}$, let $c_{n}(T)=\operatorname{dim} R\left(T^{n}\right) / R\left(T^{n+1}\right)$ and $c_{n}^{\prime}(T)=\operatorname{dim} N\left(T^{n+1}\right) / N\left(T^{n}\right)$. Then the descent of $T$ is defined by $\delta(T)=$ $\inf \left\{n: c_{n}(T)=0\right\}=\inf \left\{n: R\left(T^{n}\right)=R\left(T^{n+1}\right)\right\}$ and the ascent $a(T)$ of $T$ is defined by $a(T)=\inf \left\{n: c_{n}^{\prime}(T)=0\right\}=\inf \left\{n: N\left(T^{n}\right)=N\left(T^{n+1}\right)\right\}$.

For an element $x$ in an algebra $A$ with unit, let $L_{x}$ and $R_{x}$ denote the left and right multiplication operators by $x$. Following $[11$, p. 835] we set $a_{l}(x)=a\left(L_{x}\right), \delta_{l}(x)=\delta\left(L_{x}\right), a_{r}(x)=a\left(R_{x}\right), \delta_{r}(x)=\delta\left(R_{x}\right)$. From [11, Proposition 3.1], for each integer $n \geq 0$ we know that

$$
a_{l}(x)=\delta_{l}(x)=n \Leftrightarrow a_{r}(x)=\delta_{r}(x)=n .
$$

Moreover we know from [12, Proposition 3.2] that if $\delta_{l}(x)$ and $\delta_{r}(x)$ are both finite then $a_{l}(x)=\delta_{l}(x)=a_{r}(x)=\delta_{r}(x)$.

In [8], V. Kordula and V. Müller defined the concept of regularity as follows:

Definition 2.2. A non-empty subset $\mathbf{R} \subset A$ is called a regularity if it satisfies the following conditions:

(i) If $a \in A$ and $n \geq 1$ is an integer then $a \in \mathbf{R}$ if and only if $a^{n} \in \mathbf{R}$.

(ii) If $a, b, c, d \in A$ are mutually commuting elements satisfying $a c+b d$ $=e$, then $a b \in \mathbf{R}$ if and only if $a, b \in \mathbf{R}$.

A regularity $\mathbf{R}$ defines in a natural way a spectrum by $\sigma_{\mathbf{R}}(a)=\{\lambda \in \mathbb{C}$ : $a-\lambda I \notin \mathbf{R}\}$ for every $a \in A$. Moreover in the case of a Banach algebra $A$, the spectrum $\sigma_{\mathbf{R}}$ satisfies the spectral mapping theorem. 
Theorem 2.3. Let $A$ be an algebra with unit. Then the set $\mathbf{D R}(A)$ of Drazin invertible elements in the algebra $A$ is a regularity.

Proof. (i) Since every invertible element in $A$ is Drazin invertible, $\mathbf{D R}(A)$ is a non-empty set. Let $a \in A$ and $n \geq 1$ an integer. If $a$ is Drazin invertible, then from [5, Theorem 2], $a^{n}$ is Drazin invertible. Conversely suppose that $a^{n}$ is Drazin invertible. Then from [10, Lemma 2] there is an integer $k \geq 1$ such that $a^{n k}$ is group invertible. Again from [10, Lemma 2], $a$ is Drazin invertible.

(ii) Let $a, b, c, d$ be mutually commuting elements of $A$ such that $a c+b d$ $=e$. As proved in [5, Theorem 4], an element $a$ of $A$ is Drazin invertible if and only there are elements $c, d$ of $A$ and positive integers $p, q$ such that $a^{p}=a^{p+1} c$ and $a^{q}=d a^{q+1}$. Hence $a$ is Drazin invertible if and only if $\delta_{l}(a)$ and $\delta_{r}(a)$ are both finite. Since $a c+b d=e$, we have $L_{a} L_{c}+L_{b} L_{d}=I$ and $R_{a} R_{c}+R_{b} R_{d}=I$. From [9, Lemma 4] it follows that $\delta_{l}(a b)$ is finite if and only if $\delta_{l}(a)$ and $\delta_{l}(b)$ are finite, and $\delta_{r}(a b)$ is finite if and only if $\delta_{r}(a)$ and $\delta_{r}(b)$ are finite. Hence $a b$ is Drazin invertible if and only if $a$ and $b$ are Drazin invertible.

If $A$ is a Banach algebra with unit $e$ and if $x \in A$, we define the Drazin spectrum of $x$ by $\sigma_{\mathbf{D R}}(x)=\{\lambda \in A: x-\lambda e \notin \mathbf{D R}(A)\}$. Using the properties of regularities [8], we immediately obtain the following corollary:

Corollary 2.4. Let $A$ be a Banach algebra with unit, let $x \in A$ and let $f$ be an analytic function in a neighborhood of the usual spectrum $\sigma(x)$ of $x$ which is non-constant on any connected component of $\sigma(x)$. Then $f\left(\sigma_{\mathbf{D R}}(x)\right)=\sigma_{\mathbf{D R}}(f(x))$.

Proposition 2.5. Let $A$ be a Banach algebra with unit, let $a \in A$. Suppose that $b \in A$ is invertible and commutes with a. If a is Drazin invertible, and $b$ is sufficiently small in norm, then $a+b$ is invertible.

Proof. Suppose that $a$ is Drazin invertible. Then the bounded linear operator $L_{a}$ acting on the Banach algebra $A$ is also Drazin invertible. Hence $L_{a}$ has a finite ascent and descent. So $L_{a}$ is an operator of topological uniform descent in the sense of Grabiner [6, Definition 2.5]. Using Grabiner's punctured neighborhood theorem [2, Theorem 4.5] it follows that if $T$ is an invertible bounded linear operator commuting with $L_{a}$ and having small norm, then $c_{0}\left(T+L_{a}\right)=c_{p}\left(L_{a}\right), c_{0}^{\prime}\left(T+L_{a}\right)=c_{p}^{\prime}\left(L_{a}\right)$, for $p$ large enough. Since $a$ is Drazin invertible, for $n \geq \delta_{l}(a)$ we have $c_{n}\left(L_{a}\right)=c_{n}^{\prime}\left(L_{a}\right)=0$. So $c_{0}\left(T+L_{a}\right)=c_{0}^{\prime}\left(T+L_{a}\right)=0$ for $n \geq \delta_{l}(a)$ and this shows that $T+L_{a}$ is an invertible operator. Now if $b$ is an invertible element of $A$ commuting with $a$ and having a small norm, and if we set $T=L_{b}$, then $T$ is invertible and its norm is $\|T\|=\left\|L_{b}\right\|=\|b\|$. By the preceding argument we see that $L_{b}+L_{a}=L_{a+b}$ is invertible. So $a+b$ is invertible. 
Proposition 2.6. Let $a, b$ be two commuting Drazin invertible elements of an algebra $A$ with unit. Then ab is Drazin invertible.

Proof. It follows from [10, Lemma 2] that there is an integer $n$ such that $a^{n}$ and $b^{n}$ are group invertible. So there are $x$ and $y$ such that $x a^{n} x=x$, $a^{n} x a^{n}=a^{n}, x a^{n}=a^{n} x$ and $y b^{n} y=y, b^{n} y b^{n}=b^{n}, y b^{n}=b^{n} y$. From [5, Theorem 1], we know that $a^{n}, b^{n}, x, y$ are commuting elements. So

$$
x y(a b)^{n} x y=x y, \quad(a b)^{n} x y(a b)^{n}=(a b)^{n}, \quad(a b)^{n} x y=x y(a b)^{n} .
$$

Hence $(a b)^{n}$ is group invertible and so $a b$ is Drazin invertible.

3. An Atkinson-type theorem for B-Fredholm operators. In this part we consider the Banach algebra $L(X)$ of bounded linear operators acting on a Banach space $X$. For $T \in L(X)$, we denote by $N(T)$ the null space of $T$, by $\alpha(T)$ the nullity of $T$, by $R(T)$ the range of $T$ and by $\beta(T)$ its defect. If both $\alpha(T)$ and $\beta(T)$ are finite, then $T$ is called a Fredholm operator and the index of $T$ is defined by $\operatorname{ind}(T)=\alpha(T)-\beta(T)$. In this case it is well known that the range $R(T)$ of $T$ is closed in $X$.

For each integer $n$, define $T_{n}$ to be the restriction of $T$ to $R\left(T^{n}\right)$ viewed as a map from $R\left(T^{n}\right)$ into $R\left(T^{n}\right)$ (in particular $T_{0}=T$ ). If for some integer $n$ the space $R\left(T^{n}\right)$ is closed and $T_{n}$ is a Fredholm operator, then $T$ is called a $B$-Fredholm operator [2, Definition 2.2]. In this case from [1, Proposition 2.1], $T_{m}$ is a Fredholm operator and $\operatorname{ind}\left(T_{m}\right)=\operatorname{ind}\left(T_{n}\right)$ for each $m \geq n$. This remark leads to the following definition:

Definition 3.1. Let $T \in L(X)$ be a B-Fredholm operator and let $n$ be any integer such that $T_{n}$ is a Fredholm operator. Then the index $\operatorname{ind}(T)$ of $T$ is defined as the index of the Fredholm operator $T_{n}$.

In particular if $T$ is a Fredholm operator we get the usual definition of the index.

Let $\mathrm{BF}(X)$ be the class of all B-Fredholm operators. In [1] the first author has studied this class of operators and he has proved [1, Theorem 2.1] that $T \in L(X)$ is a B-Fredholm operator if and only if $T=Q \oplus F$, where $Q$ is a nilpotent operator and $F$ a Fredholm operator. Let us recall that an operator $T \in L(X)$ has a generalized inverse if there is an operator $S \in L(X)$ such that $T S T=T$. In this case $T$ is also called a regular operator and $S$ is called a generalized inverse of $T$. It is well known that $T$ has a generalized inverse if and only if $R(T)$ and $N(T)$ are closed and complemented subspaces of $X$. In [4], S. R. Caradus has defined the following class of operators:

Definition 3.2. $T \in L(X)$ is called a generalized Fredholm operator if $T$ is regular and there is a generalized inverse $S$ of $T$ such that $I-S T-T S$ is a Fredholm operator. 
Let $\Phi_{\mathrm{g}}(X)$ be the class of all generalized Fredholm operators. In [11], [12], C. Schmoeger has studied this class of operators and he has proved [13, Theorem 1.1] that $T \in L(X)$ is a generalized Fredholm operator if and only if $T=Q \oplus F$, where $Q$ is a finite rank nilpotent operator and $F$ is a Fredholm operator. Hence a generalized Fredholm operator is a B-Fredholm operator, but the converse is not true, for example a nilpotent operator with a non-closed range is a B-Fredholm operator but not a generalized Fredholm operator, since a non-closed range operator is not regular. Moreover the class $\mathrm{BF}(X)$ of B-Fredholm operators satisfies the spectral mapping theorem while the class $\Phi_{\mathrm{g}}(X)$ does not.

Let $A=L(X) / F_{0}(X)$ where $F_{0}(X)$ is the ideal of finite rank operators in $L(X)$ and let $\pi: L(X) \rightarrow A$ be the canonical projection. Atkinson's well known theorem [7, Theorem 6.4.3] says that $T \in L(X)$ is a Fredholm operator if and only if its projection $\pi(T)$ in the algebra $A$ is invertible. In the following result we establish an Atkinson-type theorem for B-Fredholm operators. More precisely, in a first step we prove the following important relation between B-Fredholm operators and generalized Fredholm operators in the sense of Caradus:

Proposition 3.3. Let $T \in L(X)$. Then $T$ is a B-Fredholm operator if and only if there exists a positive integer $p \in \mathbb{N}$ such that $T^{p}$ is a generalized Fredholm operator.

Proof. If $T$ is a generalized Fredholm operator, then $T$ is a B-Fredholm operator. Conversely if $T$ is a B-Fredholm operator, then from [1, Theorem 2.1], $T=Q \oplus F$, where $Q$ is a nilpotent operator and $F$ a Fredholm operator. Let $n$ be an integer such that $Q^{n}=0$. Then $T^{n}=Q^{n} \oplus F^{n}=0 \oplus F^{n}$. Since $F^{n}$ is a Fredholm operator, from [13, Theorem 1.1] we see that $T^{n}$ is a generalized Fredholm operator.

Theorem 3.4. Let $T \in L(X)$. Then $T$ is a B-Fredholm operator if and only if $\pi(T)$ is Drazin invertible in the algebra $L(X) / F_{0}(X)$.

Proof. From [11, Theorem 3.3] it follows that $T$ is a generalized Fredholm operator if and only $\pi(T)$ is group invertible in the algebra $L(X) / F_{0}(X)$. Using the preceding proposition we see that $T$ is a B-Fredholm operator if and only if there exists $p \in \mathbb{N}$ such that $\pi\left(T^{p}\right)$ is group invertible. Since $\pi\left(T^{p}\right)=\pi(T)^{p}$, using [10, Lemma 2] we see that $T$ is a B-Fredholm operator if and only if $\pi(T)$ is Drazin invertible in $L(X) / F_{0}(X)$.

Corollary 3.5. (i) Let $T_{1}, T_{2}$ be B-Fredholm operators such that $T_{1} T_{2}$ and $T_{2} T_{1}$ are finite rank operators. Then $T_{1}+T_{2}$ is a B-Fredholm operator.

(ii) Let $T_{1}, T_{2}$ be commuting B-Fredholm operators. Then $T_{1} T_{2}$ is a $B$ Fredholm operator. 
(iii) Let $T$ be a B-Fredholm operator and let $F$ be a finite rank operator. Then $T+F$ is a B-Fredholm operator.

Proof. Let $A=L(X) / F_{0}(X)$ and let $\pi: L(X) \rightarrow L(X) / F_{0}(X)$ be the canonical projection. Then $\pi$ is an algebra homomorphism and:

(i) We have $\pi\left(T_{1}\right) \pi\left(T_{2}\right)=\pi\left(T_{2}\right) \pi\left(T_{1}\right)=0$. From [5, Corollary 1] it follows that $\pi\left(T_{1}+T_{2}\right)=\pi\left(T_{1}\right)+\pi\left(T_{2}\right)$ is Drazin invertible in $A$. So $T_{1}+T_{2}$ is a B-Fredholm operator.

(ii) We have $\pi\left(T_{1} T_{2}\right)=\pi\left(T_{1}\right) \pi\left(T_{2}\right)=\pi\left(T_{2}\right) \pi\left(T_{1}\right)$. From Proposition 2.6 it follows that $\pi\left(T_{1} T_{2}\right)$ is Drazin invertible. Hence $T_{1} T_{2}$ is a B-Fredholm operator.

(iii) If $F$ is a finite rank operator and $T$ is a B-Fredholm operator then $\pi(T+F)=\pi(T)$. So $T+F$ is a B-Fredholm operator.

REMARK. The class of B-Fredholm operators is not stable under compact perturbations, that is, $\mathrm{BF}(X)+K(X) \nsubseteq \mathrm{BF}(X)$ where $K(X)$ is the closed ideal of all compact operators in $L(X)$. For example let $\left(\lambda_{n}\right)_{n}$ be a sequence in $\mathbb{C}$ such that $\lambda_{n} \neq 0$ for all $n$ and $\lambda_{n} \rightarrow 0$ as $n \rightarrow \infty$, and consider the operator $T$ defined on the Hilbert space $l^{2}(\mathbb{N})$ by

$$
T\left(\xi_{1}, \xi_{2}, \xi_{3}, \ldots\right)=\left(\lambda_{1} \xi_{1}, \lambda_{2} \xi_{2}, \lambda_{3} \xi_{3}, \ldots\right) .
$$

Then

$$
T^{n}\left(\xi_{1}, \xi_{2}, \xi_{3}, \ldots\right)=\left(\left(\lambda_{1}\right)^{n} \xi_{1},\left(\lambda_{2}\right)^{n} \xi_{2},\left(\lambda_{3}\right)^{n} \xi_{3}, \ldots\right)
$$

Since $\left(\lambda_{m}\right)^{n} \neq 0$ for all $m \geq 0$ and $\left(\lambda_{m}\right)^{n} \rightarrow 0$ as $m \rightarrow \infty$ for all $n \geq 0$ we see that $T^{n} \in K(X)$ and $T^{n}$ is not a finite rank operator for all $n \geq 1$. Hence $R\left(T^{n}\right)$ is not closed for all $n \geq 1$. Thus $T \notin \operatorname{BF}(X)$. Since $0 \in \operatorname{BF}(X)$ it follows that $\mathrm{BF}(X)+K(X) \nsubseteq \mathrm{BF}(X)$.

As a consequence of this remark, if $\mathbf{C}(X)=L(X) / K(X)$ is the Calkin algebra and if $\Pi: L(X) \rightarrow \mathbf{C}(X)$ is the canonical projection, then $\Pi(T)=0$ is Drazin invertible in $\mathbf{C}(X)$ but $T$ is not a B-Fredholm operator.

Open Questions. We finish this paper by the following open questions, suggested by a comparison between Fredholm operators and B-Fredholm operators:

1. It is well known that if $S, T$ are Fredholm operators, then $S T$ is a Fredholm operator and $\operatorname{ind}(S T)=\operatorname{ind}(S)+\operatorname{ind}(T)$, where ind is the index. Now if $S, T$ are commuting B-Fredholm operators, we know from Corollary 3.5 that $S T$ is a B-Fredholm operator. Is it still true that $\operatorname{ind}(S T)=\operatorname{ind}(S)+\operatorname{ind}(T)$ ?

2. Let $T$ be a Fredholm operator and $K$ a compact operator. It is known that $T+K$ is a Fredholm operator and $\operatorname{ind}(T+K)=\operatorname{ind}(T)$. Now if $T$ is a B-Fredholm operator and $F$ a finite rank operator, then $T+F$ is a B-Fredholm operator. Do we have ind $(T+F)=\operatorname{ind}(T)$ ? 


\section{References}

[1] M. Berkani, On a class of quasi-Fredholm operators, Integral Equations Oper. Theory 34 (1999), 244-249.

[2] - Restriction of an operator to the range of its powers, Studia Math. 140 (2000), 163-175.

[3] M. Berkani and M. Sarih, On semi-B-Fredholm operators, Glasgow Math. J., to appear.

[4] S. R. Caradus, Operator Theory of the Pseudo-Inverse, Queen's Papers in Pure and Appl. Math. 38, Queen's Univ., Kingston, Ont., 1974.

[5] M. P. Drazin, Pseudo-inverses in associative rings and semigroups, Amer. Math. Monthly 65 (1958), 506-514.

[6] S. Grabiner, Uniform ascent and descent of bounded operators, J. Math. Soc. Japan 34 (1982), 317-337.

[7] R. Harte, Invertibility and Singularity for Bounded Linear Operators, Dekker, 1988.

[8] V. Kordula and V. Müller, On the axiomatic theory of spectrum, Studia Math. 119 (1996), 109-128.

[9] M. Mbekhta and V. Müller, On the axiomatic theory of spectrum II, ibid. 119 (1996), 129-147.

[10] S. Roch and B. Silbermann Continuity of generalized inverses in Banach algebras, ibid. 136 (1999), 197-227.

[11] C. Schmoeger, On a class of generalized Fredholm operators, I, Demonstratio Math. 30 (1997), 829-842.

[12] -, Ascent, descent and the Atkinson region in Banach algebras, I, Ricerche Mat. 42 (1993), 123-143.

[13] -, On a class of generalized Fredholm operators, V, Demonstratio Math. 32 (1999), 595-604.

Département de Mathématiques

Faculté des Sciences

Université Mohammed I

Oujda, Maroc

E-mail: berkani@sciences.univ-oujda.ac.ma
Département de Mathématiques

Faculté des Sciences

Université Ibn Tofail

Kénitra, Maroc

Received July 3, 2000

Revised version April 10, 2001 